\title{
CLUSTERS OF TOURISM CONSUMERS IN ROMANIA
}

\author{
Associate Professor Corina Pelau PhD, \\ Bucharest University of Economic Studies, Romania \\ E-mail: corinapelau@yahoo.com \\ Alexandra Catalina Chinie \\ Bucharest University of Economic Studies, Romania
}

\section{(Received: January 2018; Accepted March 2018)}

\begin{abstract}
The analysis and determination of typologies of tourism consumers has been a major concern for scientists, specialists and companies as well. Knowing the demographic and motivational factors that determine consumers to buy tourism products can have a major impact on the marketing strategy by a more efficient targeting of customers. This article presents the results of a research that aims to determine the factors which influence the buying decision for tourism products and the clusters of consumers resulted from these factors. 90 persons have been surveyed pursuing the determination of the most important factors for buying a tourism product and the correlation between them. The factor analysis and the cluster analysis have been applied with the help of the SPSS program. The results of the factor analysis group the items into six factors. In a second phase, the consumers have been divided into three categories based on a hierarchical Ward cluster analysis. The three clusters have been defined and analyzed and recommendations for the future research have been given.
\end{abstract}

Key words: Consumer, tourism products, factor analysis, cluster analysis, buying decision J.E.L. CODES: M31

\section{Introduction}

The researchers in the fields of consumer behavior and tourism have tried for many years to explain the buying decisions of consumers for tourism products. Depending on this behavior a tourism company can adjust its marketing strategy and it can influence their decision. As Solomon et al. (2010) mention, without understanding the way the consumer of tourism products feels and thinks, it is almost impossible for a company to offer the products he wants and in the way he wants them. This is amplified in the recent years by the fact that the bought products don't represent just a way of spending the spare time anymore, but they also describe the social position and status of the consumer. Consequently, the consumer buys the products to define his image, express his personality and consolidate his image in the society (Solomon, et al. 2010, p. XIV). Knowing the behavior and the complex factors which drive the consumer to buy products help tourism companies to develop their strategies. For a company it is important to 
determine both the demographic and the motivational factors that determine consumers to have a certain buying behavior.

The objective of this article is to determine the factors that influence the buying decision of tourism products and to determine clusters of tourism consumers based on their prefences. The article presents the results of a factor analysis regarding the grouping of the analyzed itmes and the results of a cluster analysis carried out based on the previously determined factors.

\section{Literature review}

Due to its economic and social importance, tourism has been studied from various disciplinary points of view (Benckendorff \& Zehrer, 2013). Studying the consumer behaviour has been one of the most frequent studies in the field of tourism. In their review of the tourism consumer behavior body of knowledge Cohen et al. (2014) identified the key concepts, influences and research contexts analyzed in the three mainstream tourism journals. Among the most studied topics were satisfaction, trust and loyalty, motivations and perceptions of tourists and perceptions, the influence of technology on the field and the context of cross-cultural issues in emerging markets (Cohen et al., 2014). In the marketing field, analyzing the factors that influence the consumer behavior of people that travel and their motivation is relevant to establishing strategies aimed at specific target groups.

One of the main concerns of consumer behavior in general and specific for tourism products is the segmentation and the determination of clusters of consumers with similar motivations, preferences and behavior. Knowing the motivation of different groups of consumers can help companies target them better and hereby improve their strategy (Pop \& Pelau, 2007).

Li et al. (2013) have studied the influence that being part of a certain generation has on information source preference, visitation history, destination preferences, destination evaluation criteria and travel activity preferences while grouping the surveyed population into four generational groups. Age categories have been analyzed in other similar studies that researched the tourism consumer behaviour (Losada et al., 2016; Fyall et al., 2017) and the general consumer behavior (Serban et al. 2017). Similarly, behavioral differences between genders have been approached by researchers (Hudson, 2000). According to Tkaczynski et al. (2009), variables that were most often used to profile tourists were behavioural, geographic, demographic and psychographic (trip purpose and motivations).

In his study, Chen (2003) classified tourists into four unique segments, where two of them were considered actionable - with a higher probability to make recommendations, and two were non-actionable. Differences between the two 
actionable groups were triggered by the education level and willingness to join a group tour (Chen, 2003). Legoherel et al. (2015) identified tourist groups based on their variety-seeking behavior, proposing a new way of segmentation for the international travelers market.

\section{Research methodology}

The objective of the research presented in this article is to determine the factors that influence the buying decision of tourism products and especially the influence of reference groups (friends, family and relatives) on the buying decision. The research was conducted in April-May 2014 achieving 90 valid responses. The sample contains an even number of men and women and is homogeneously distributed among age groups. In order to determine the factors influencing the buying decision, a questionnaire has been created, with Likert scale questions from 1 to 5 , where 5 represents a very strong influence and 1 represents a small influence. The research results were determined and analyzed in two phases. The first phase was the factor analysis, followed by a cluster analysis.

The factor analysis in SPSS has been applied, in order to determine the factors influencing the buying decision. The factor analysis deals with the grouping of elements which influence the dependent variable, based on the correlations among them. Of course if there are a small number of elements, the regression analysis can be used, but if you increase the number of independent variables it is recommended to use the factor analysis (Backhaus, et al., 2000, pg. 253). For the analysis presented in this article, 48 items have been chosen, which may influence the buying decision of tourism products. The aim of the factor analysis was to reduce the number of items based on the correlations among them. In order to determine the groups of factors, the factor analysis was run twice as presented in the following paragraph.

In the first stage the factor analysis was conducted without imposing a number of factors. Applying the Kaiser-Meyer-Olkin criterion, we obtain an MSA value (measure of sampling adequacy) of 0.525 that displays acceptably suitable criteria for this analysis. The values of the main diagonal of the anti-image matrix show an acceptable adequacy of the items described. The item with an under average adequacy are the importance of tourist attractions, price, distance from home, cultural diversity, interest for a particular location, and interest for cruises and seaside.

Based on this type of factor analysis, taking in consideration the eigenvalues greater than one, an ideal number of 12 factors have been indicated. However, if we look at the loadings of the 48 items on the 12 factors, it can be observed that six factors have a small number of associated items. Besides, a look at the scree-plot 
and the elbow criterium shows an adequate number of six factors. Therefore, we resume the factor analysis by defining a fix number of factors. It is worth mentioning that the 12 factors could explain $76.9 \%$ of the variance of the buying decision.

The factor analysis is run for the second time, by indicating the number of six factors. It is done both for 48 items and also for 41 items with a high adequacy (the items with a low adequacy mentioned above, are eliminated). For the reduction of the 48 items with the help of the factor analysis we obtained an MSA value of 0.525 , while the same analysis applied to the 41 items obtains an MSA value of 0.629. Since the loading of the factor is similar for both cases, we are going on with the results of the factor analysis applied to the 48 items. The result of the analysis of the component matrix and the loadings of the factors can be observed in Annex 3. Since there are no big differences, we continue with the analysis of the 48 items. These factors explain 58.8 of the total variance of the dependent variable and of the buying decision.

The cluster analysis was run with the purpose of determining homogenous groups of consumers, with similar buying behavior regarding tourism products. The cluster analysis was run for the factors determined with the help of the factor analysis. The first aspect determined with the help of the cluster analysis is the optimal number of clusters. The recommended instrument for the determination of the number of clusters is the elbow-criterion. Because of the fact that there was no "elbow" in the Ward procedure, showing a rather high homogeneity of the cluster member, the dendogram was analyzed. Based on the dendogram, 3 clusters were determined. With the help of the SPSS program the cluster membership of all respondents has been determined.

In order to analyze the characteristics and the adequacy of the clusters, the F-test and t-test for the cluster analysis were used. The F-test is an indicator used for the measure of the homogeneity of the factors within a cluster. It is calculated as the relation between the variance of the analyzed factor (variable) within a cluster and the variance of the factor/ variable within the whole sample (Backhaus, et al. 2000, pg. 378). The t-test is used for the characterization of the clusters, measuring the over- or under representation of a factor. It is measured as the relation between the difference between the average value of the factor/variable in the cluster and the average value of the factor/ variable for the whole sample related to the standard deviation of the factor/ variable in the whole sample (Backhaus, et al. 2000, pg. 379). Based on the values of the factors and their F-test and t-test, the clusters were characterized. The clusters were named after the main characteristics, taking preponderantly in consideration the items with the highest homogeneity. 


\section{Results of the factor analysis}

As we have seen in the previous chapter, the factor analysis determined six factors that influence the buying decision for tourism products. The factor analysis has been applied by using the SPSS program. Figure 1 shows the distribution of the 48 items on the six determined factors. This factor loading can also be observed in Annex 3, which presents the result of the analysis.

\begin{tabular}{|c|c|c|}
\hline FACTOR 1 & FACTOR 2 & FACTOR 3 \\
Market trends & Tourist attractions & Popularity of destination \\
Options of colleagues & Tourist objectives & Options of friend \\
Opinions of colleagues & Degree of quietness of the & Opinion of friends \\
Weather conditions & destination \\
Pollution at destination & Internet offers & Press information \\
TV information & Information tourist agencies & Newsletter \\
Social networks & Interest for a certain location & Ambiance \\
Position of the hotel & Clubs and coffee-shops & Health treatments \\
Number of hotel stars & Cultural diversity & \\
Quality of food & Organized tours & \\
Possibility of making sport & Relaxation & \\
Spa treatments & Visiting of objectives & Culture \\
Shopping & Social & \\
City Break & Fun & \\
Cruises & Outdoor activities & FACTOR 6 \\
Mountains & FACTOR 5 & Uniqueness of location \\
\hline FACTOR 4 & Options of relatives & Internet information \\
\hline Celebration of events & Opinion of relatives & \\
Interest for location & Distance to home & \\
Exotic destinations & Seaside &
\end{tabular}

Figure 1 Item loadings on the six factors

As it can be seen in figure 1, there is a greater number of items assigned to the first three factors and a smaller number of items on the last factors. This can be explained by a double elbow, once after the third factor and once after the sixth factor. In fact, in a less precise analysis, three factors can be analyzed. But because we want a more detailed analysis, in this article we considered the distribution on six factors.

Basically the items within the same factor have a similar influence on the consumer. Therefore, a consumer who responds to one item of the factor in a certain way will react to the other items in a similar way. Besides, the items are parts of one factor and if one of them is not met, it affects the whole item. Therefore, for a better customer satisfaction it is important to know all the elements 
that affect a certain factor.

By analyzing the content of the items of each factor, the following notations can be considered for each of the factors.

\section{Factor 1: Offered conditions (2.91)}

The first factor was called „offered conditions" because it contains items such as number of stars of the hotel, position of the hotel, food quality and other general conditions such as weather, pollution level, etc. As it can be seen in Figures 2a and $2 \mathrm{~b}$, the items that define this factor have a great importance for the consumer. We have items that analyze the conditions related to the touristic product, such as position of the hotel (3.33), number of stars of the hotel (3.42), food quality (3.93), all of them having values higher than 3 (rather important). The general conditions have also a great importance for the consumer. Items such as the weather conditions (3.63) and the degree of pollution of the destination (2.40) have a similar effect on the consumer. If the weather conditions have an important influence on the consumer, they are less aware and give less importance to the pollution at the destination.

As it can be seen in figures $2 \mathrm{a}$ and $2 \mathrm{~b}$, in addition to the factors related to accommodation and the general conditions, in this factor there are also included items, related to the possibility of fun and leisure. This factor includes the opportunity to do sport (2.35), spa treatments (2.31) and shopping (2.95). None of these factors has a great importance for the consumer, but they are associated to general conditions offered by a touristic package.

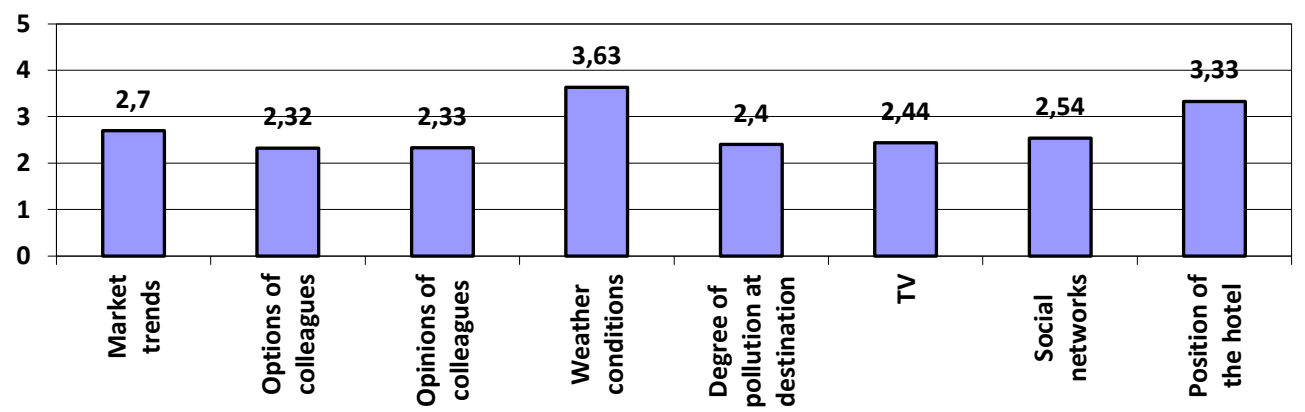

Figure 2a Characteristic of items in factor 1 


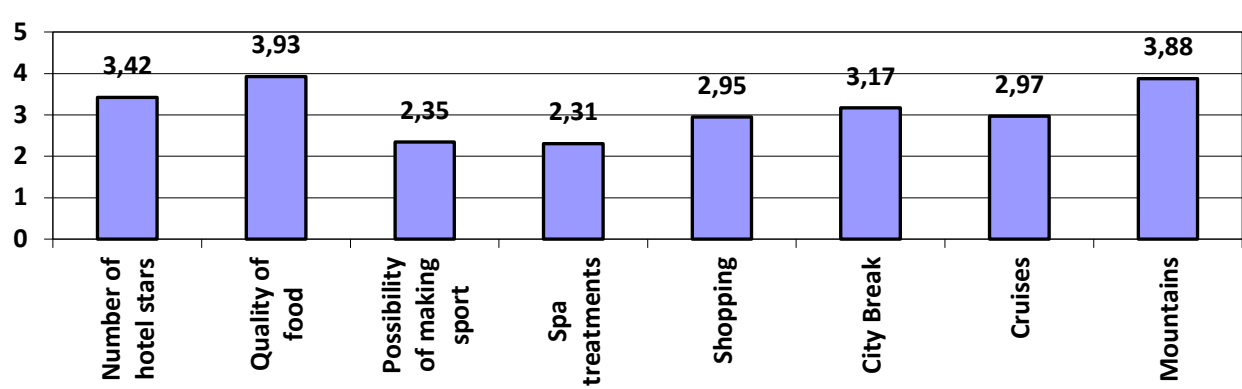

Figure 2b Characteristic of items in factor 1

Regarding the reference group, this factor is associated with the opinions and options of colleagues. The relationship between these items and the factor can be understood in several ways. On one hand, the decision of the colleagues might affect the consumer's decision, as he buys the same products as his colleagues. Therefore, a consumer buys a tourism product at the recommendation or by imitating a colleague, knowing that in this way he obtains the desired conditions. On the other hand, the association of this item with the factor "offered conditions" can be interpreted in the sense that the consumer chooses a destination with a certain standard in order not to be outdone by his colleagues. Anyway, it's interesting to observe that the reference groups' colleagues, friends and relatives are associated with various factors.

Another category of items associated with this factor are city breaks (3.17), cruises (2.97) and going to the mountains (3.88). The consumer associates the good conditions offered by travelling to certain destinations such as visiting cities (citybreak), cruises and trips to the mountains. He can expect good conditions at the mentioned destinations or he travels to these destinations because they offer him the desired conditions. It is interesting to observe that these destinations have greater importance for the consumer than the general conditions.

Items such as the market trends, destinations seen on TV or social networks are also included in this factor. Therefore, the consumer creates some expectations based on seen media, and collects its information from them.

\section{Factor 2: Attraction degree of the destination (3.62)}

The second factor was named „the attraction degree of the location” based on the items that have been associated to it. As it can be seen in the value indicated in the title, it has a greater importance for the consumer than the first factor. This factor was named in this way because it contains items related to the attractivity of the 
Pelau C., Chinie A.C. (2018)

Clusters of tourism consumers in Romania

location, the sights and attractions, but also other factors such as the presence of culture, existence of clubs and cafes, the possibility of interaction through social activities.

The items with the highest importance for the consumer are those related to the attrativity of the destination, as shown in figures $3 \mathrm{a}$ and $3 \mathrm{~b}$. Among these, there are the existence of tourist attractions (4.30), tourist sights (4.34), sightseeing (3.92) and interest for a specific destination (4.08). In fact these items, with the exception of sightseeing, have the highest value in the whole research. These items related to the specificity of the location are complemented by other elements such as the presence of cultural activities (3.67), cultural diversity (3.62) possibility of outdoor activities (3.41), the existence of clubs and cafes (2.73) and the possibility of interaction with the social environment (2.90).

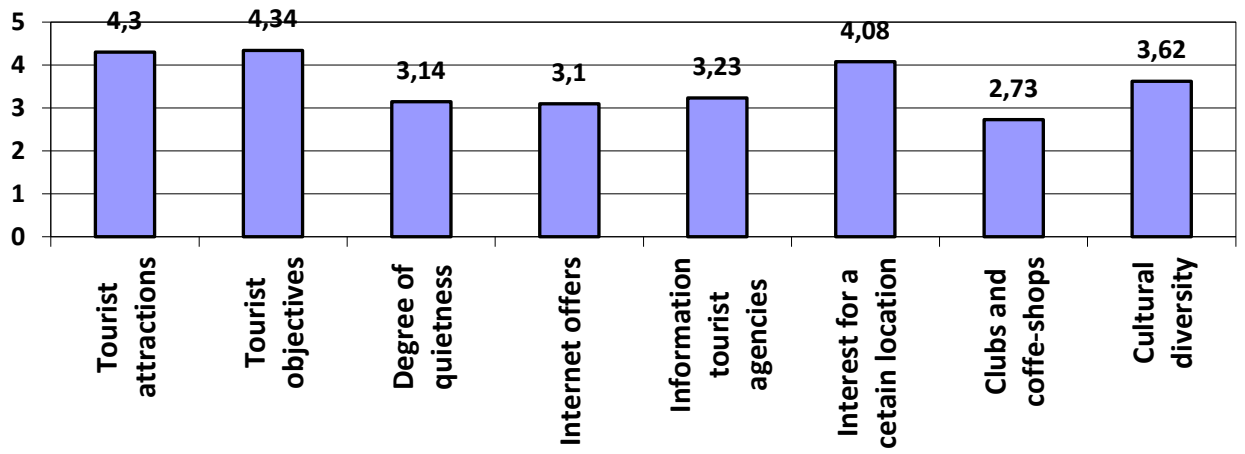

Figure 3a Characteristic of items in factor 2

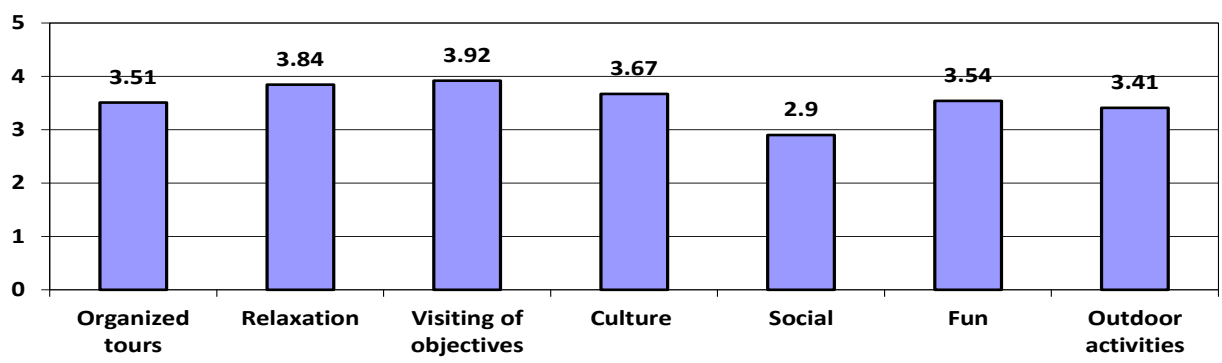

Figure 3b Characteristic of items in factor $2(2)$

In contrast to the first factor, which focused on the materialistic part of the 
conditions offered, this factor contains the general conditions related to the emotional part such as the ability to relax (3.84), entertainment (3.54) and the degree of quietness of the location (3.14), all of them having an importance above the average.

It is interesting to observe that this factor does not contain elements related to the reference groups, but focuses more on personal issues like the interest for a destination or its quietness. Thus, consumers choose as a source of information the offers on the internet (3.10) or information from the travel agencies (3.23). Both forms of communication have greater importance in comparison with other items. It's interesting to observe that from all types of packages, this factor contains the organized tours. Consumers mostly associate the activity of visiting of various sights with the participation in organized tours. It can be seen in figure $3 \mathrm{~b}$ that organized tours (3.51) have an above average importance for the consumers.

\section{Factor 3: Popularity and utility of the destination (2.98)}

The third factor is entitled ,popularity and utility of the destination”, containing a variety of items. The most important factors for consumers are the price (3.96) and the ambiance (3.91). The high importance of the price is the item that gives the name of utility to the factor. Another element related to the utility is the presence of health treatment facilities (2.12), which also suggest a practical purpose.

An important element of this factor is the popularity (3.33). Although apparently it is not directly related to the price, the consumer has a similar behavior for the two items. Ambience (3.91) can also complete the item popularity, giving the name of this factor.

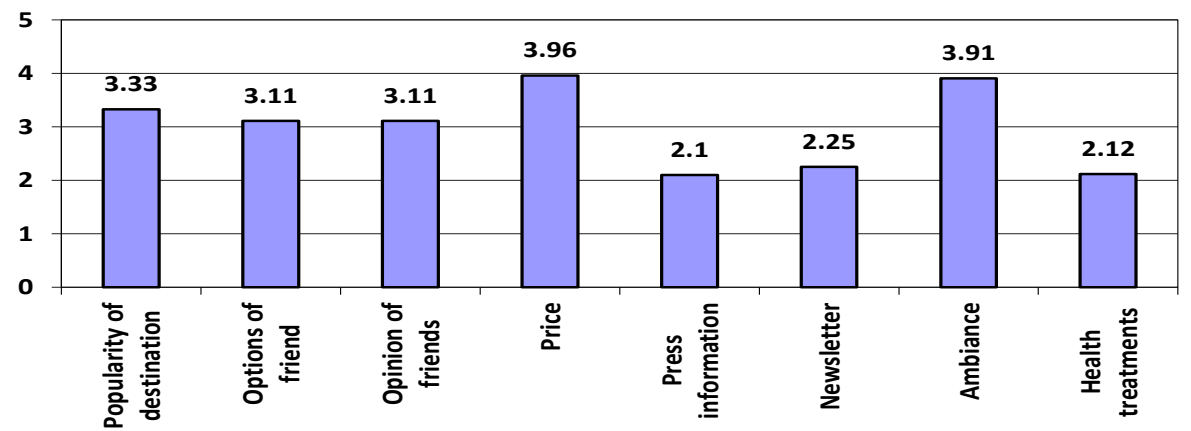

Figure 4 Characteristic of items in factor 3

DE GRUYTER OPEN
Studia Universitatis "Vasile Goldis" Arad. Economics Series Vol 28 Issue 1/2018 ISSN: 1584-2339; (online) ISSN: 2285 - 3065

Web: publicatii.uvvg.ro/index.php/studiaeconomia.Pages $17-34$ 
Another interesting result from the factor analysis is the relation of the options and opinions of friends to this factor. The relationship between friends and this factor can also have multiple interpretations. On one hand, the consumer can be influenced by friends in terms of price and popularity of destination. On the other hand, the consumer can choose a specific tourism product to impress his/her friends. Products are chosen with a high popularity at similar prices to those paid by the group of friends. The marketing communication means that influence the buying decisions of the products associated to this factor best are the newsletter and the press information.

The next presented factors are defined by a smaller number of items. As mentioned earlier in this article, the scree-plot shows an ideal number of three or six factors. For a better accuracy the set with six factors has been chosen for this article. Probably in another form of the factor analysis, the following factors would be assimilated into the first three.

\section{Factor 4: Exotic character of the location (3.55)}

This factor contains four items, namely the celebration of events, the interest for a location, great exotic destinations and the seaside. From all mentioned factors, the most important one is the desire to go to the seaside (4.06).

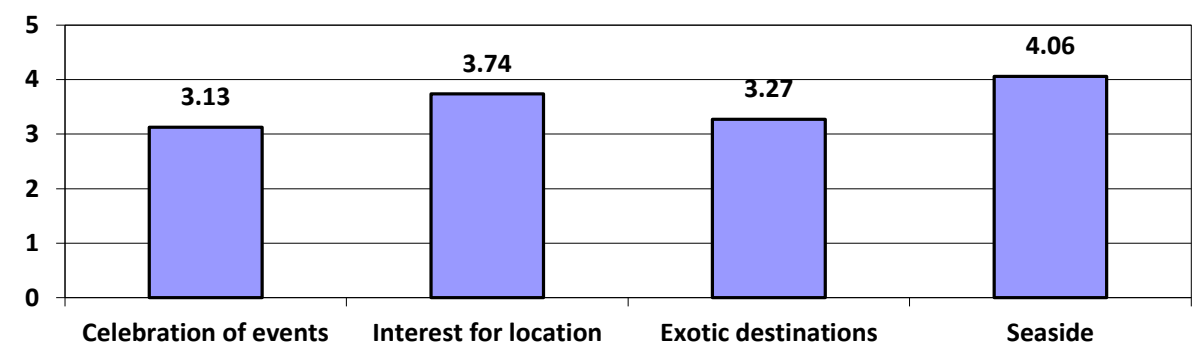

Figure 5 Characteristic of items in factor 4

The interest in a particular location (3.74) also has a high importance. It seems that the desire to go to the seaside is a good enough reason to buy a tourism product, with this destination. The item exotic destination (3.27) has a great importance. In fact in most cases, the seaside is a destination of this kind. The destinations for holiday are also included in this factor. In this case the desire to spend the holidays 
in a certain location is a reason enough to buy a tourism product.

\section{Factor 5: The influence of the family and personal issues (2.34)}

This factor contains three items related to family and the proximity to it. As it can be seen in figure 6 , none of the three items has a great importance. The item with the highest value is the option of the relatives (2.42), followed by the distance to home (and implicitly to the relatives) (2.37) and the relatives opinion (2.23).

Of all reference groups, the relatives seem to have the least influence, fact confirmed also by other research. It is important to observe, that in contrast to friends and colleagues, there is a difference between the opinion of the relatives and their options. Although with a small importance, the relatives option is more important than their opinion, suggesting an imitative behavior.

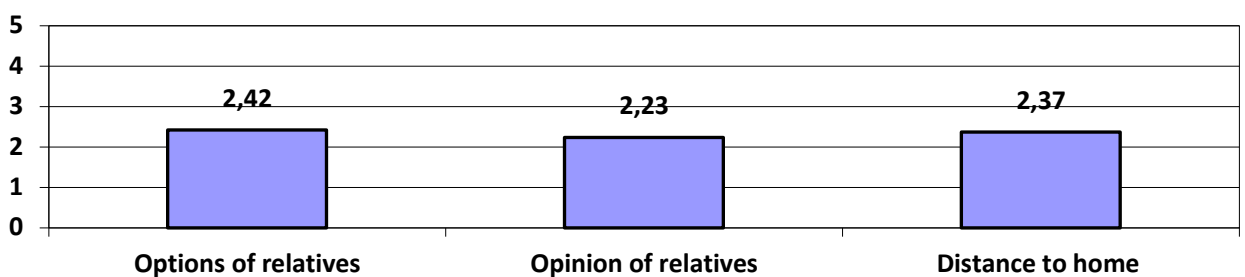

Figure 6 Characteristic of items in factor 5

\section{Factor 6: Uniqueness of the location (3.75)}

The last factor contains only two items with a high importance for the consumer. The most important item for this factor is the uniqueness of the location (4.08) and the information from the internet. The consumer is determined to buy a tourism product based on the uniqueness of a destination.

The link to the item internet information can be made through the internet communication. Therefore the consumer can find information about unique locations mainly on the internet.

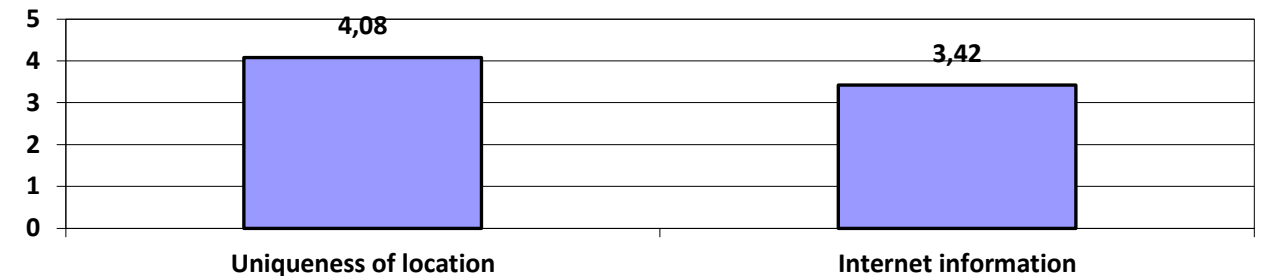

Figure 7 Characteristic of items in factor 6

DE GRUYTER OPEN
Studia Universitatis "Vasile Goldis" Arad. Economics Series Vol 28 Issue 1/2018 ISSN: 1584-2339; (online) ISSN: 2285 - 3065

Web: publicatii.uvvg.ro/index.php/studiaeconomia.Pages $17-34$ 
If we look at the average values of their importance, we can observe that the factor uniqueness of the location (3.75) and the attraction degree of the location (3.62) are the most interesting for the consumer. A great impact on the buying decision has also the exotic character of the location (3.55). The offered conditions (2.91) and the popularity and utility of the destination (2.98) have apparently an average impact. Lower values of these two factors may be due to the large number of items contained by them. It is possible that these factors may contain items with a low attractiveness but which were best suited here. Family and personal items have the slightest influence on the decision of buying a tourism product.

\section{Results of the cluster analysis}

In order to determine the typologies of consumers of tourism products, a cluster analysis was run for the factors determined in the previous chapter. As we saw in the methodology description, the elbow criteria didn't show a clear delimitation of the cluster, showing that there is a homogeneous behavior for tourism products. Analyzing the dendogramm of the cluster analysis, three clusters can be observed. Therefore, the cluster analysis ran for a fixed number of three clusters.

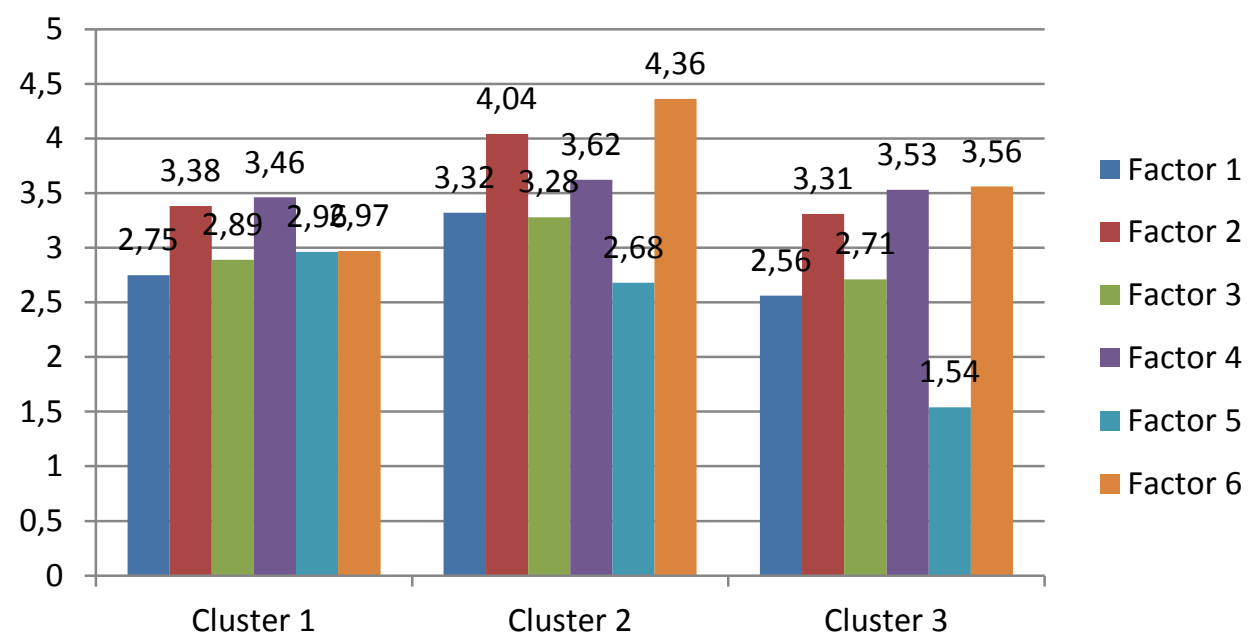

Figure 8 Characteristics of the tourism consumer clusters

Figure 8 depicts the characteristics of the three tourism consumer clusters, based on the values of the six analyzed factors. There are factors that are similar for the three clusters as for instance factor 4 - the exotic character of the location and factors which are different and which show the characteristic of the cluster. For instance 
factor 6 - uniqueness of the location is the main factor for cluster 2 and cluster 3 . Similarly factor 2 - attraction degree of the destination and factor 4 - exotic character of the location have higher values for all three clusters showing again the priorities for each of the clusters. Factor 3 - popularity and utility of the destination and factor 1 - offered conditions are higher for cluster 2, while factor 5 - influence of the family and personal issues is higher for cluster 1.

Based on these results the three clusters were defined as follows:

Cluster 1: Attraction seekers

Cluster 2: High-value and uniqueness seekers

Cluster 3: Independent uniqueness seekers

\section{Cluster 1: Attraction seekers}

This cluster was defined based on the factors with the highest values, which are the exotic character of the location (3.46) and the attraction degree of the destination (3.38). Analyzing the F-test for these factors it can be observed that the attraction degree of the destination (0.11) has the highest homogeneity among the cluster members. Therefore the cluster received its name after this predominant factor. In opposition to this, the factor "exotic character of the location” has the highest value for the F-test showing the highest heterogeneity.

For this cluster it is important to point out the fact that factor 5 - influence of family and personal issues (2.96) plays an important role for its representatives as it has the highest value in the clusters. In opposition to this, factor 6 - the uniqueness of the location has the lowest value in the cluster -2.97 . Both factors have an average value for the F-test of 0.36 and 0.38 showing an average homogeneity. The positive value of the T-test for factor 5 (0.71) shows a higher importance of this factor for the cluster in comparison to the whole sample. The opposite is valid for the other 5 factors.

This cluster is predominantly feminine, as $52.4 \%$ of its representatives are female. In opposition to the other two cluster, most of the people belonging to this cluster are older than 40 years. So, $57,1 \%$ of the cluster members are older than 40 years, while only $42.9 \%$ of its members are younger than 40 years. According to the marital status, this is the cluster with the highest percentage of married people or people living in a couple as only $33.3 \%$ are single. Analysing these characteristics, it can be stated that it is a mature cluster, including mostly settled members, with a richer life experience.

\section{Cluster 2: High-value and uniqueness seekers}

This cluster was actually named based on the high value for the offered conditions, showing that for this category of customers the conditions play an important role in 
the buying decision. This factor has a value of the F-test of 0.78 showing a medium homogeneity of the members of this cluster. In contrast, the factors with the highest values are the uniqueness of the location (4.36) and the attraction degree of the destination (4.04). While the factor 6 - uniqueness of the location shows the highest homogeneity for the cluster (0.43), factor 2 has the highest heterogeneity (1.39). It is also the cluster for which factor 3 - popularity and utility of the destination has the highest value, having also a medium heterogeneity of 0.72 . The results of the t-test shows a higher importance of the factors for this cluster than for the other clusters.

Cluster 2 is also a predominantly feminine cluster, but in opposition to cluster 1 it is a rather young cluster. $54.1 \%$ of its members are females and $67,6 \%$ of its members are younger than 40 years. Most of the people in this cluster are married (48.6\%), while $10.8 \%$ live together without being married. Analyzing the characteristics of the cluster it can be observed that young people have higher value expectations than older people, as a cluster oriented towards value has a rather young membership.

\section{Cluster 3: Independent uniqueness seekers}

Cluster 3 was defined based on the item with the lowest value (1.54) of factor 5 the influence of the family and personal issues. This is also the factor which has the highest homogeneity according to the F-test (0.19).

The items which have the highest values for this factor are the uniqueness of the location (3.56), the exotic character of the location (3.53) and the attraction degree of the destination (3.31). This cluster has also the lowest value for the offered conditions (2.56) and popularity and utility of the location (2.71). All these elements show the independence of the tourists. With the exception of factor $4-$ exotic character of the location, which has a value of 1.15 in the F-test, all the other factors have an adequate homogeneity. Regarding the impact of the factors, all of them have an under average influence on the group in comparison to the whole sample. This is indicated by the negative values of the t-test.

It is the only predominantly masculine cluster, as $56.3 \%$ of its members are males. Analyzing the characteristics of the group and the masculinity, a match can be observed as it is known that males are more individualistic than females. It is also a rather young cluster, $61.1 \%$ of its members being younger than 40 years. Similar to cluster $2,56.3 \%$ of the members of the clusters are married or live in a couple, while the rest are single.

In spite of the fact, that most of the factors contain different types of representatives, some tendencies can be still observed. Therefore, two of the clusters are preponderantly feminine and one of them is mainly masculine. 
Regarding the age, one of the clusters has mainly members older than 40 years, while 2 of them have members younger than 40 years.

\section{Conclusions}

As it is well known a research can not reveal all aspects of a certain field, but it can contribute to the knowledge in a certain domain. Especially in a complex field such as the decision to buy a tourism product, which involves besides the satisfaction with the product several aspects related to social issues and status, there is a need for a detailed analysis.

A first result of the factor analysis shows that the items chosen in the research are powerful and can be selected as independent factors to a large extent. However, it is difficult to make an analysis based on over 40 variables, which is why the multivariate method factor analysis is applied. Analyzing the distribution and loading of items, six factors can be identified.

The variance obtained from the factor analysis reveals that the factors do not fully cover the reasons why consumers buy tourism products. Although a large number of influence factors are present (as items), the buying decision is more complex. Thus other factors can be determined in future with the help of exploratory research. It is interesting to observe that the influence of the reference groups (family, friends, colleagues) fall under three different factors, showing that they are treated as different aspects of the tourism product. Colleagues are included in the first factor, which describes the general conditions, indicating that colleagues are a source of information for the offered conditions. Friends are included in the popularity factor, having two possible interpretations. On one hand, consumers can consider the destinations visited by their friends to be popular, so that the options and opinions of friends go into this factor. Another interpretation of this grouping may be the fact that consumers choose popular destinations in order to have a good image at their friends. Besides, from all reference groups, friends have the greatest influence (3.11), followed by colleagues (2.33) and relatives (2.23). The influence of relatives is small and it is interesting to note that the options of the relatives have a greater influence on the buying decision than their opinion, showing an imitation of the behavior rather than its importance.

The results of the cluster analysis show that for the Romanian population it is difficult to divide the tourism consumers in precise clusters. This is shown first by the results of the scree plot, which did not have a clear elbow. Therefore, the multivariate analysis did not show a clear delimitation of clusters, indicating similarities among each members. Secondly, if we analyze the characteristics of the clusters, again similarities can be found. Another aspect is the fact that, the results of the t-test show a higher importance of the factors for the second cluster, than for

Studia Universitatis "Vasile Goldis" Arad. Economics Series Vol 28 Issue 1/2018 
the other ones. Therefore, there might be also other factors influencing the buying decision of the consumers. Consequently, a further exploratory study is recommended. The only element indicating a difference between the clusters is the F-test. For cluster 1 and cluster 3, one factor can be identified, which has a high homogeneity (probably the factor which defines the cluster), all the others having an average homogeneity. Cluster 2 is the only one with average homogeneity for most factors, including probably all members who didn't fit in the other two clusters). Despite this, factor 2 is the one to have the highest representation of factors in comparison to the whole sample. Factor two is also the biggest cluster having the highest number of members.

Consequently, it can be stated that the Romanian consumers are influenced by several factors, without having a certain pattern in their behavior. One reason for this behavior is the communist era where because of the system, most of the consumers had the same behavior. Therefore, the tourism companies have a difficult task in trying to develop targeted strategies to such a heterogeneous consumer market. Despite of this, some factors can still be observed, which have a great importance for the consumer. It is recommended to implement mass-oriented strategies, using the factors which the consumers find interesting such as the uniqueness and attraction degree of the location or its exotic character. Having such an "unpatterned" consumer he will probably respond easily to marketing instruments, being a topic for future research.

\section{References}

1. Backhaus, K.; Erichson, B.; Plinke, W.; Weiber, R. (2000), Multivariate Analysemethoden - Eine anwendungsorientierte Einfuehrung, Springer, Berlin.

2. Benckendorff, P. and Zehrer, A. (2013), A network analysis of tourism research, Annals of Tourism Research, Vol. 43, pp. 121-149.

3. Chen, J.S. (2003), Market segmentation by tourists' sentiments, Annals of Tourism Research, Vol. 30, Issue 1, pp. 178-193.

4. Cohen, S.A., Prayag, G. And Moital, M. (2014), Consumer behaviour in tourism: Concepts, influences and opportunities, Current Issues in Tourism, Vol. 17, Issue 10, pp. 872-909.

5. Fyall, A., Leask, A., Barron, P., Ladkin, A. (2017), Managing Asian attractions, Generation Y and face, Vol. 32, pp. 35-44.

6. Hudson, S. (2000), The segmentation of potential tourists: contraint differences between men and women, Vol. 38, pp. 363-368.

7. Legoherel, P., Hsu, C. H.C., Daucé, B. (2015), Variety-seeking: Using the CHAID segmentation approach in analyzing the international traveler market, Tourism Management, Vol. 46, pp. 359-366. 
8. Li, X. Li, X.R. and Hudson, S. (2013), The application of generational theory to tourism consumer behavior: An American perspective, Tourism Management, Vol. 37, pp. 147-164.

9. Losada, N., Alén, E., Domínguez, T., Nicolau, J. L. (2016), Travel frequency of seniors tourists, Tourism Management, Vol. 53, pp. 88-95.

10. Pop, N. Al.; Pelau, C. (2007), Knowing the motivation of buying, in: Analele universităţii din Oradea, seria: Ştiinţe economice, editura Universităţii din Oradea, Tom XVI, 2007, pg. 1063-1066.

11. Tkaczynski, A., Rundle-Thiele, S.R., Beaumont, N. (2009), Segmentation: A tourism stakeholder view, Tourism Management, Vol. 30, pp. 169-175.

12. Serban, D.; Pelau, C.; Chinie, A.C. (2017), Calculation of the Differences of Attitude and Behavior of two Generations with the Euclidian-Metric, in Proceedings of the 11th International Conference on Business Excellence, pg. 241-247.

13. Solomon, M.; Bamossy, G.; Askegaard, S.; Hogg, M. K. (2010), Consumer Behavior A European Perspective, 4th edition, Prentice Hall, Financial Times, Harlow.

Annex 1 : F-test values for the cluster analysis

\begin{tabular}{|l|r|r|r|r|r|r|}
\hline \multicolumn{7}{|c|}{ F test } \\
\hline & Factor_1 & \multicolumn{1}{|c|}{ Factor_2 } & Factor_3 & Factor_4 & Factor_5 & Factor_6 \\
\hline Cluster 1 & 0.807 & 0.119 & 0.714 & 1.649 & 0.368 & 0.380 \\
\hline Cluster 2 & 0.780 & 1.396 & 0.725 & 0.536 & 0.876 & 0.439 \\
\hline Cluster 3 & 0.590 & 0.396 & 0.582 & 1.150 & 0.198 & 0.513 \\
\hline
\end{tabular}

Annex 2 : t-Test values for the cluster analysis

\begin{tabular}{|l|c|c|c|c|c|c|}
\hline \multicolumn{7}{|c|}{ T test } \\
\hline & Factor_1 & Factor_2 & Factor_3 & Factor_4 & Factor_5 & Factor_6 \\
\hline Cluster 1 & -0.25459 & -0.37202 & -0.20718 & -0.14462 & 0.719135 & -1.04381 \\
\hline Cluster 2 & 0.634115 & 0.617855 & 0.670284 & 0.115314 & 0.392245 & 0.816001 \\
\hline Cluster 3 & -0.56638 & -0.47047 & -0.63924 & -0.03849 & -0.92523 & -0.25861 \\
\hline
\end{tabular}

Studia Universitatis "Vasile Goldis" Arad. Economics Series Vol 28 Issue 1/2018 ISSN: 1584-2339; (online) ISSN: 2285 - 3065 
Pelau C., Chinie A.C. (2018)

Clusters of tourism consumers in Romania

\begin{tabular}{|c|c|c|c|c|c|c|}
\hline & \multicolumn{6}{|c|}{ Component } \\
\hline & 1 & 2 & 3 & 4 & 5 & 6 \\
\hline Popularity of destination & .407 & -.183 & .583 & -.069 & -.050 & -.232 \\
\hline Market trends & .551 & -.165 & .347 & -.271 & .032 & -.258 \\
\hline Options of friends & .225 & -.212 & .486 & -.369 & .433 & .157 \\
\hline Opinions of friends & .300 & -.204 & .547 & -.281 & .330 & .162 \\
\hline Tourist attraction & .025 & .328 & .171 & 188 & .035 & .561 \\
\hline Uniqueness of location & .079 & .339 & .399 & .043 & 266 & .419 \\
\hline Option of colleagues & 692 & .104 & .257 & -.237 & .382 & .114 \\
\hline Opinions of colleagues & .692 & .017 & .230 & -.255 & .419 & -.081 \\
\hline Tourist objectives & -.181 & .506 & -.008 & .325 & .208 & .426 \\
\hline Quieteness of destination & .316 & .596 & -.081 & -.057 & -.458 & -.035 \\
\hline Weather conditions & .513 & .336 & .174 & -.135 & -.250 & .168 \\
\hline Price & -.084 & -.097 & -.580 & .016 & .107 & .429 \\
\hline Options of relatives & .384 & .382 & -.351 & -.150 & .523 & -.173 \\
\hline Opinions of relatives & 400 & .406 & -.262 & -.086 & .564 & -.164 \\
\hline Degree of pollution & .588 & .232 & -.468 & -.124 & -.171 & .133 \\
\hline Distance to home & .318 & .078 & -.393 & -.351 & -.348 & .214 \\
\hline Press information & .529 & .005 & -.542 & -.085 & -.124 & .170 \\
\hline Internet offers & .128 & -.426 & .003 & -.309 & -.161 & .459 \\
\hline Tourism agency information & .392 & .448 & -.021 & -.180 & -.111 & -.293 \\
\hline TV information & .760 & -.173 & -.347 & -.305 & -.121 & -.001 \\
\hline Internet information & .270 & -.358 & 193 & -.258 & -.211 & .509 \\
\hline Newsletter & .398 & -.304 & -.471 & -.178 & -.066 & .267 \\
\hline Social networks & .559 & -.520 & .268 & .066 & .035 & .098 \\
\hline Interest for destinaton & .193 & .529 & .221 & .215 & .172 & -.036 \\
\hline Hotel position & .572 & .329 & .457 & -.055 & -.322 & -.168 \\
\hline Number of hotel stars & .642 & .201 & .341 & -.136 & -.330 & -.273 \\
\hline Existance of clubs/coffee shops & .523 & -.620 & .088 & .097 & -.036 & .068 \\
\hline Ambiance & .326 & .216 & .506 & 221 & -.230 & .114 \\
\hline Quality of food & .488 & .219 & .118 & .353 & -.359 & -.130 \\
\hline Exotic destinations & .214 & -.066 & .075 & .670 & .050 & -.163 \\
\hline Cultural diversity & .173 & .364 & -.049 & .340 & 278 & .289 \\
\hline Organized tours & .160 & 645 & .042 & -.013 & -.026 & .123 \\
\hline Relaxation & .279 & .452 & -.008 & .360 & -.276 & -.221 \\
\hline Visiting of objectives & .489 & .505 & -.199 & .139 & -.061 & .339 \\
\hline Culture & .436 & .584 & -.065 & .085 & -.061 & .333 \\
\hline Celebration of events & .347 & -.296 & -.216 & .366 & -.109 & -.135 \\
\hline Social & .497 & -.560 & -.288 & -.064 & .010 & -.078 \\
\hline Sport & .628 & -.376 & -.223 & .183 & .085 & -.061 \\
\hline Interest for location & .064 & .315 & .003 & .323 & .253 & .140 \\
\hline Health treatments & .239 & .268 & -.505 & -.025 & .193 & -.275 \\
\hline Spa treatments & .443 & .279 & -.373 & -.070 & .295 & -.227 \\
\hline Shopping & .421 & -.379 & -.286 & .390 & .108 & -.217 \\
\hline City break & .535 & -.366 & .128 & .262 & -.140 & .144 \\
\hline Cruises & .420 & .178 & .284 & .372 & .160 & -.036 \\
\hline Fun & .224 & -.647 & .005 & .405 & .337 & -.009 \\
\hline Outdoor activities & 429 & -.529 & -.087 & .413 & .048 & .203 \\
\hline Mountains & .326 & -.319 & -.121 & 268 & -.125 & .042 \\
\hline Seaside & -.147 & -.246 & .201 & .281 & -.168 & .187 \\
\hline
\end{tabular}

DE GRUYTER OPEN
Studia Universitatis "Vasile Goldis" Arad. Economics Series Vol 28 Issue 1/2018 ISSN: 1584-2339; (online) ISSN: 2285 - 3065

Web: publicatii.uvvg.ro/index.php/studiaeconomia.Pages 17 - 34 